\title{
Cauda Equina Syndrome: A Case Report
}

\author{
T. Hari keerthana ${ }^{1 *}$, Vijay Jagadeesh Kakimani ${ }^{1,}$ N. Surendra Reddy ${ }^{1}$, \\ Kali Chandrasekhar ${ }^{2}$, D. Ranganayakulu ${ }^{3}$ \\ ${ }^{I}$ Department of Pharmacy Practice, Sri Padmavathi School of Pharmacy, Tirupati, AP, India. \\ ${ }^{2}$ Assistant Professor, Department of General Medicine, Sri Venkateshwara Medical College, Tirupati, AP, \\ India. ${ }^{3}$ Principal Sri Padmavathi School of Pharmacy, Tiruchanoor, Tirupati-517503, India.
}

\begin{abstract}
Cauda equina syndrome (CES) is a rare syndrome which is characterized by low back pain, unilateral or bilateral sciatica, motor weakness of lower extremities, saddle anesthesia, bowel and bladder dysfunction and occasionally paraplegia - resulting from compression of the cauda equina. It can be caused by herniated disc, tumor, infection, fracture, or narrowing of spinal cord. It can be described in two forms based on the onset of signs and symptoms. if symptoms appear within 24hours then it is acute in onset and if patient develop symptoms after several weeks or months then it is gradual in onset. The sooner it can be diagnosed, the better chance the patient makes a better recovery from symptoms of nerve damage. It generally requires prompt surgical decompression in order to decrease or eliminate pressure on the affected nerves. surgical decompression is advocated as soon as possible, within about 8 hours of the onset of symptoms if symptoms develop suddenly. If patients with cauda equina syndrome do not receive treatment quickly, adverse results can include paralysis, impaired bladder, and/or bowel control, difficulty walking, and/or other neurological and physical problems.
\end{abstract}

Keywords: CES, bladder dysfunction, nerve damage, MRI, surgical decompression.

\section{Introduction:}

Cauda equina syndrome (CES) is a rare syndrome that has been described as a complex of symptoms and signs - low back pain, unilateral or bilateral sciatica, motor weakness of lower extremities ${ }^{1}$, saddle anesthesia, bowel and bladder dysfunction and occasionally paraplegia ${ }^{2}$-resulting from compression of the cauda equina. It is a medical emergency that needs for urgent surgical intervention. CES may be caused by a herniated disc, tumor, infection, fracture, or narrowing of the spinal canal ${ }^{3}$. It is of two types based on onset; acute and gradual. If symptoms occur within 24hours then it is acute in onset and if patient develop symptoms after several weeks or months then it is gradual in onset. MRI (magnetic resonance imaging) is the standard method of confirming the presence of CES and for planning surgical treatment. The sooner it can be diagnosed, the better chance the patient makes a better recovery from symptoms of nerve damage. It generally requires prompt surgical decompression in order to decrease or eliminate pressure on the affected nerves. surgical decompression is advocated as soon as possible, within about 8 hours of the onset of symptoms if symptoms develop suddenly. Next to surgery, the extent of recovery is uncertained. Patients may continue to experience low back or leg pain, bladder or bowel dysfunction, and other physical problems depending on the duration of nerve compression and the severity of symptoms at the time of surgery. If patients with cauda equina syndrome do not receive treatment quickly, adverse results can include paralysis, impaired bladder, and/or bowel control, difficulty walking, and/or other neurological and physical problems ${ }^{4}$. This article discusses a case report of CES with late diagnosis and the permanent harm encountered by the patient.

\section{Case Report:}

A 45 years old male patient admitted or presented to the general medicine male department of SVRRGGH, Tirupati with the complaints of weakness, decreased sensation and reduced cloth sensation of both lower limbs which are gradually progressive in nature. Patient's past medical history revealed that he had low back pain, radicular type which is more on right side and is asymmetric in onset. He had involuntary urination since 6months. At present he has developed ulcer on buttock region as he is bed ridden due to his inability to walk. His laboratory data include Hb- $8 \mathrm{~g} / \mathrm{dl}$, PLT- $1.7 \mathrm{ks} / \mathrm{cumm}$, Serum creatinine- $0.9 \mathrm{mg} / \mathrm{dl}$, Serum electrolytes: $\mathrm{Na}^{+}-137 \mathrm{mmol} / 1, \mathrm{~K}^{+}-3.8 \mathrm{mmol} / 1, \mathrm{Cl}^{-}-92 \mathrm{mmol} / \mathrm{l}$. MRI scan had shown disc dehydration with annular tear at L5-S1 level, diffuse bulge at L4-5, L5-S1levels, narrowing the spinal canal, neural foramina on left, intending the thecal sac. These features are in the favour of "Spinal cord AV malformation". Based on the complaints, past medical history and MRI scan of the patient he was diagnosed with "Cauda Equina Syndrome". Later he was treated symptomatically with Tab. paracetamol 500mg orally thrice daily, Tab. B-complex orally once daily, Tab. cefixime $200 \mathrm{mg}$ orally twice daily, Tab. Pantoprazole $40 \mathrm{mg}$ orally once daily, Tab. Metrogyl $400 \mathrm{mg}$ orally thrice daily, Inj. Tramadol 100mg IM twice daily and Inj. Optineuron IM once daily. After that the 
patient did not improve the symptoms and was suggested to get further DSA evaluation but the patient was not in the state to afford the test and left the hospital.

\section{Discussion}

In patients with low back pain, the presence of one of the following features - saddle anesthesia, urinary retention or incontinence, bowel incontinence, sensory or motor weakness in either of the lower extremities - indicates the diagnosis of $\mathrm{CES}^{5}$. The most common cause for CES is lumbar disc prolapse (1-10\% of cases), it can also be caused by trauma, spinal canal stenosis, tumours, epidural haematoma/abscess, inferior vena cava thrombosis and spinal manipulation ${ }^{6}$. Two features make cauda equina susceptible to the effects of compressive and tensile forces. First, the nerve roots have no Schwann cell covering; second, cauda equina lacks a regionalised segmental blood supply with relative hypovascularity in the central portion of the nerve root, making it vulnerable to ischaemia from compression ${ }^{7}$.

The standard presentation of CES includes low back pain with saddle anaesthesia, bilateral or unilateral lower limb sensory and motor disturbances and bowel/bladder involvement ${ }^{8}$. Firstly, our patient was presented with lower back pain along with symptoms of saddle anesthesia which are progressive in nature. The lower back pain was more on right side which is sudden in onset. initially the patient was diagnosed as chronic inflammatory demyelinating polyneuropathy (CIDP) and had taken treatment symptomatically and discharged from the hospital. Then the same patient visited the hospital after 6months with same complaints along with urinary retention and bed sores. Contrary to this, several case series have been reported with varied clinical manifestations like unilateral leg symptomatology 8,9 , saddle anesthesia with or without leg symptoms and CES with complete absence of signs and symptoms in the lower limbs. (young,8, olaire,9). O'Laoire reported CES with acute bladder retention and lack of sensory deficit in 2 out of his 29 patients ${ }^{10}$.

The patient was sent to MRI scan immediately and the reports had shown the disc dehydration with annular tear at L5-S1 level, diffuse bulge is seen at L4-5 and L5-S1 levels and then narrowing of the spinal canal and neural foramina on the left side was observed which is intended towards thecal sac. the above features are suggestive of spina lcord AV malformation and the patient was recommended with further digital subtraction angiography (DSA) evaluation. The doctors thought that they need DSA evaluation to operate the patient. unfortunately it is not available to the patient in our hospital and the patient is also not in the state to afford the test. After that the patient was discharged.

If the patient was diagnosed early for CES signs it would have helped the patient to improve signs and symptoms through operation. But the patient was diagnosed lately after 6months. Therefore, misdiagnosis and procedural delay are also expensive causes of problems faced by the patient.

\section{Conclusion}

This case is presented for its characteristic clinical presentation of a dehydrated disc and narrowing of spinal canal and neural foramina causing compression of the cauda equina producing bilateral loss of sensation and motor power with bladder involvement. These are the premonitory features of CES and failure to recognize these symptoms and signs can delay the diagnosis of CES by the orthopedic surgeon. This can result in patient morbidity such as loss of bladder, bowel and sexual function. Therefore, it is important that all orthopaedic surgeons should have a detailed knowledge of the clinical presentations of this condition and assess the patients with low back pain for signs of CES. The prognosis of this condition rely on early diagnosis, as timely surgical interference before bladder bowel involvement will give satisfying functional recovery and the quality of life of the patient can be improved.

\section{References}

[1]. Alex Gitelman, MD, Shuriz Hishmeh, MD, Brian N. Morelli, MD, Samuel A. Joseph, Jr., MD, Andrew Casden, MD, Paul Kuflik, MD, Michael Neuwirth, MD, and Mark Stephen, MD; Cauda Equina Syndrome: A Comprehensive Review; The American Journal of Orthopedics.

[2]. ER Crowther, BA, DC; Slow onset cauda equina syndrome:a case report; 0008-3194/93/203-209/\$2.00/@JCCA 1993.

[3]. http://orthoinfo.aaos.org/topic.cfm?topic=a00362.

[4]. http://www.spine-health.com/conditions/lower-back-pain/treatments-cauda-equina-syndrome.

[5]. Balaji Douraiswami, M.S. (Ortho), D.N.B. (Ortho), M.Ch. (Orth), Kumanan Muthuswamy, M.S. (Ortho), Dilip Kumar Naidu, M.S. (Ortho), M.Ch. (Orth), Sriram Thanigai, M.S. (Ortho), and Vijay Anand, M.S. (Ortho); Indeterminate cauda equina syndrome: A case report; 2016 Jan-Mar; 7(1): 50-54. Published online 2015 Jul 18.

[6]. Ma B., Wu H., Jia L.-s., Yuan W., Shi G.-d., Shi J.-g. Cauda equina syndrome: a review of clinical progress.Chin Med J. 2009;122(10):1214-1222.

[7]. Rydevik B.L. Neurophysiology of cauda equina compression. Acta Orthop Scand. 1993;64(suppl 251):52-55.

[8]. Kostuik J.P., Harrington I., Alexander D., Rand W., Evans D. Cauda equina syndrome and lumbar disc herniation. J Bone Joint Surg Am. 1986;68(3):386-391.

[9]. Scott P.J. Bladder paralysis in cauda equina lesions from disc prolapse. J Bone Joint Surg Br. 1965;47:224-235.

[10]. O'Laoire S., Crockard H.A., Thomas D.J.G. Prognosis for sphincter recovery after operation for cauda equina compression owing to lumbar disc prolapse. BMJ. 1981;282:1852-1854. 